\title{
Latent variable and network models of comorbidity: toward an empirically derived nosology
}

\author{
Nicholas R. Eaton
}

Received: 4 December 2014/ Accepted: 8 January 2015/Published online: 20 January 2015

(C) Springer-Verlag Berlin Heidelberg 2015

\begin{abstract}
Although current classification systems, such as the diagnostic and statistical manual of mental disorders (DSM-5) [1], define mental disorders as putatively independent phenomena, comorbidity is the rule rather than the exception. Indeed, nearly half of individuals meeting criteria for one mental disorder will meet criteria for at least one more [2]. Through bivariate analyses, we can note that disorders such as major depressive disorder (MDD) and generalized anxiety disorder (GAD) overlap more frequently than expected by chance alone [3-5]. Based on their prevalence rates, data from one nationally representative sample indicated four individuals per 1,000 should have comorbid MDD and GAD; however, 17 such casesmore than $400 \%$ of the expected frequency-were observed [5].
\end{abstract}

High rates and diffuse patterns of comorbidity suggest fundamental problems with current nosologies. To many, they also represent foci for classification research in that understanding the nature of comorbidity may help characterize the fundamental, transdiagnostic building blocks of psychopathology and thereby reconceptualize psychiatric constructs. Thus, framing comorbidity as a meaningful subject of inquiry in itself, rather than as a failure of classification systems that should be remedied, presents an exciting opportunity to understand what mental disorder really is. Ideally, by bootstrapping from imperfect nosologies, we can iteratively improve the validity of our diagnostic constructs and eventually produce a close fit between the model (nosology) and the data (observed manifestation of psychopathology in individuals).

N. R. Eaton $(\square)$

Department of Psychology, Stony Brook University,

Stony Brook, NY 11794, USA

e-mail: nicholas.eaton@stonybrook.edu

\section{Latent variable models of comorbidity}

For the past few decades, researchers have applied a variety of latent variable models to characterize comorbidity among signs and symptoms of given disorders as well as among diagnostic entities themselves. These models are based in the same factor analytic tradition that yielded the $g$ factor of intelligence [6] and big five personality domains [7-9]. Put simply, these models posit that the observed associations among measured variables can be thought of as consequences of one or more latent (unobserved) variables [10]. These latent variables form the basis of the common factor model: Various tests that tap into different types of intelligence relate positively to one another because they are all saturated by a latent $g$ factor, and personality questionnaire items about interest in talking to people, going to parties, and so on relate positively to one another because they are all saturated by a latent extraversion factor.

When applied to diagnostic data, latent variable modeling approaches suggest latent factors that account for observed multivariate comorbidity among disorders. Early applications of this approach to psychopathology data indicated that children's symptoms and behaviors tended to covary broadly in two fundamental ways, suggestive the presence of two latent variables: internalizing and externalizing $[11,12]$. Subsequent application in adult psychopathology data $[13,14]$ led to a proliferation of latent variable modeling studies replicating this structure [1522], where the internalizing latent variable represents comorbidity among unipolar mood and anxiety disorders, and externalizing represents comorbidity among substance use disorders and various impulsivity-, oppositionality-, and antisociality-related disorders.

Latent variable modeling of these comorbidity factors has proven quite scientifically generative. These latent 
variables have been shown to be largely genetic in origin [23], but they also can be impacted by environmental exposures, such as childhood maltreatment and victimization and harassment experiences [24, 25]. They appear across numerous nationalities and cultures [22], and they are invariant across age [26], gender [16, 21, 27], race/ ethnicity [20], and sexual orientation [25]. They account for the development of lifetime comorbidity and continuity of disorders over time [18, 26, 28]. They relate closely to personality traits [29], and they account for the links between disorders and important outcomes, such as suicide $[28,30]$. They are also a growing focus of potentially effective and efficient interventions-by treating the underlying core of multiple disorders, it is hoped that intervention could have a diffuse effect [31, 32].

\section{Network models of comorbidity}

In the past several years, a different conceptualization of comorbidity - the network model-has been presented [33-35]. A compelling new direction, network model proponents largely reject the latent variable explanations of comorbidity in favor of the notion that mental disorders are composed of networks of causally connected symptoms. Some of these symptoms cause symptoms in other disorders' networks, and, together, can characterize a broad network of associations among disorders and thus comorbidity. This network approach accounts for comorbidity without many of the assumptions of the latent variable model, including the presence of higher-level latent comorbidity factors that cause observed comorbidity. For instance, network models do not assume that a disorder is measured or indicated by its symptoms, which is a foundation of the latent variable model; rather, the network approach says that the symptoms themselves are "connected through a dense set of strong causal relations" [33]. By not assuming that a latent disorder variable causes all manifested symptoms of a disorder, dynamic flexibility is introduced. For instance, while a latent variable model would indicate that MDD symptoms of sleep disturbances and fatigue were both largely caused by the latent depression variable, the network model could account more easily for the possibility that the sleep disturbances directly caused the fatigue, and that this causal observation does not require an unobserved variable to account for it [33].

Application of network models suggests that half of the symptoms of DSM-IV are connected in a network fashion [35], providing an alternate view of comorbidity - wherein particular symptoms cause one another dynamically, and where disorders are linked by symptoms that bridge their networks - that does not require broad latent variables. Rather than MDD and GADs observed comorbidity representing manifestations of a latent internalizing variable, proponents of network models argue that this comorbidity is thus due to direct causal links among symptoms. A result is a visual depiction of the symptom network, which illustrates the pattern and strength of associations among symptoms as well as the centrality of a particular symptom to a given network.

This network model of comorbidity turns analysis of comorbidity on its head, eliciting both supportive and critical feedback from scholars. While some researchers resonate with the novelty and promise of network models [36-38], others claim that network model proponents compare their models to strawman latent variable models [39] and suggest we know more than we do about observed phenomena [40]. Because network comorbidity models can include a great number of parameters, they have been criticized for their lack of parsimony and thus perceived as unlikely to uncover basic organizational structures that can become the focus of scientific inquiry [41]. This allegation stands in contrast to latent variable models, which reduce dimensionality of a space and thus produce investigable constructs such as internalizing, which, for instance, has subsequently been shown to be nearly perfectly correlated with trait neuroticism [29].

\section{Toward an empirically derived nosology}

The latent variable and network model conceptualizations of comorbidity have been framed as something of a competition, wherein researchers in one camp challenge the methodology of the other. In my view, it is obvious that both models have strengths and limitations. Thus, the question is not whether we should use latent variable or network models; the question is how best to use both to inform nosology. Both approaches represent potentially potent tools in the psychopathologist's toolkit, and, as has been noted, latent variable models can be thought of as a class of network models [42]. Even in light of their limitations, latent variable models have proven to be highly generative in advancing theory and, with the organizational meta-structure of DSM-5 reflecting these latent variables and treatments being developed to target them, they influence classification, assessment, and practice as well. While there are concerns that network models, due to their general lack of parsimony and comparatively more difficult interpretation, may not be as generative [41], the potential of this new class of models to inform research likely remains to be fully realized. From Achenbach's pioneering work $[11,12]$ with children, it took decades for the latent variable model of comorbidity to take hold firmly, and only time will tell if network models are as informative in classification research as they have been for other diverse 
topics, like neural systems, power grids, and World Wide Web structure [33].

In this era of NIH's Research Domain Criteria, issues of classification and construct refinement are increasingly coming to the fore as researchers attempt to link psychiatric phenotypes with biological substrates. Latent variable and network modeling approaches represent key players in this endeavor, particularly insofar as they can highlight core psychiatric phenomena that account for observed symptomatological and diagnostic comorbidity or identify central, highly connected nodes, respectively. Reliable and valid phenotypes can serve as targets for molecular genetic and functional imaging analyses, among others, and both modeling approaches to comorbidity appear potentially highly informative for classification (Johnson).

If the goal is to determine the core constructs of psychiatry and develop an empirically derived nosology, the more modeling options researchers have at their disposal, the better. These are thus exciting times in classification research, for several reasons. First, the proliferation of multiple new types of latent variable models-for instance, those incorporating latent class and latent trait structures simultaneously [28, 43]—allow new options for testing latent structure, particularly given the possible taxonic distributions of some disorders [44]. These variable-centered analyses, coupled with person-centered approaches such as model-based cluster analysis, hold great potential.

Second, a focus on causality, including using time-sensitive longitudinal modeling approaches, may be highly informative [45]. While neither latent variable or network models can demonstrate causality directly [46], thoughtful use of both models can highlight areas likely causal pathways. An increased focus on well-characterized longitudinal psychopathology data will also help adjudicate possible causal links, as can be seen by recent time-sensitive survival-type analysis of person-years in the development of lifetime comorbidity [18, 47]. Incorporating approximated counterfactuals, through structural equation [48-50] and novel co-twin control analyses [51-53], within these comorbidity models will also likely prove beneficial in understanding causality.

Finally, construct refinement does not need to focus solely on the level of diagnoses. Rather, symptom-level analyses in latent variable and network approaches [33, 43, 54] can be highly informative. Indeed, this appears to be an area in which the measurement focus of latent variable models might be highly compatible with the network approach, wherein imperfectly measured symptoms could be treated as latent variables with multiple indicators [55]. The pathways among, and relative importance of, these latent symptom variables could be assessed through a network modeling approach.

\section{Summary}

Latent variable models of comorbidity have significantly advanced the field of psychopathology and have proven highly generative in both research and applied contexts. Network models are relatively new in comorbidity research, and they have yet to receive a similar degree of application. While frequently framed as competing models, there is no reason to believe any single model will definitively capture comorbidity patterns and refine psychiatric nosology. In tandem with other analytic advances, however, these approaches can likely highlight key constructs at the observable and latent levels, which can be used to bootstrap from imperfect nosologies to more valid ones, iteratively. All models have strengths and weaknesses. Building upon a common quotation [56], all psychopathology models are wrong, and certainly incomplete, but some of them are useful. Thus, rather than asking which tool is best for all purposes, comorbidity and classification researchers should ask which tool is most appropriate for a given purpose-and how these sophisticated tools can complement one another to capture phenomena as complex as mental disorder.

Conflict of interest The author states that there is no conflict of interest.

\section{References}

1. American Psychiatric Association (2013) Diagnostic and statistical manual of mental disorders, vol 5. American Psychiatric Publishing, Arlington

2. Kessler RC, Chiu WT, Demler O, Walters EE (2005) Prevalence, severity, and comorbidity of 12-month DSM-IV disorders in the National Comorbidity Survey Replication. Arch Gen Psychiatry 62(6):617-627. doi:10.1001/archpsyc.62.6.617

3. Krueger RF, Markon KE (2006) Reinterpreting comorbidity: a model-based approach to understanding and classifying psychopathology. Annu Rev Clin Psychol 2:111-133

4. Mineka S, Watson D, Clark LA (1998) Comorbidity of anxiety and unipolar mood disorders. Annu Rev Psychol 49:377-412

5. Eaton NR, South SC, Krueger RF (2010) The meaning of comorbidity among common mental disorders. In: Millon $\mathrm{T}$, Krueger R, Simonsen E (eds) Contemporary directions in psychopathology: Scientific foundations of the DSM-V and ICD-11, 2nd edn. Guilford Publications, New York, pp 223-241

6. Spearman C (1904) "General intelligence", objectively determined and measured. Am J Psychol 15:201-293

7. Costa PT, McCrae RR (1992) Revised NEO Personality Inventory (NEO-PI-R) and NEO Five-Factor Inventory (NEO-FFI) manual. Psychological Assessment Resources, Odessa

8. Digman JM (1989) Five robust trait dimensions: development, stability, and utility. J Pers 57(2):195-214

9. Saucier G, Goldberg LR (1996) The language of personality: lexical perspectives on the five-factor model. In: Wiggins JS (ed) The five-factor model of personality: theoretical perspectives. Guilford, New York, pp 21-50 
10. Thurstone LL (1947) Multiple-factor analysis. University of Chicago Press, Chicago

11. Achenbach TM, Edelbrock CS (1978) The classification of child psychopathology: a review and analysis of empirical efforts. Psychol Bull 85(6):1275-1301

12. Achenbach TM, Edelbrock CS (1984) Psychopathology of childhood. Annu Rev Clin Psychol 35:227-256

13. Krueger RF (1999) The structure of common mental disorders. Arch Gen Psychiatry 56:921-926. doi:10.1001/archpsyc.56.10. 921

14. Krueger RF, Caspi A, Moffitt TE, Silva PA (1998) The structure and stability of common mental disorders (DSM-III-R): a longitudinal-epidemiological study. J Abnorm Psychol 107(2):216-227. doi:10.1037/0021-843X.107.2.216

15. Vollebergh WAM, Iedema J, Bijl RV, de Graaf R, Smit F, Ormel J (2001) The structure and stability of common mental disorders: the NEMESIS study. Arch Gen Psychiatry 58(6):597-603. doi:10.1001/archpsyc.58.6.597

16. Eaton NR, Keyes KM, Krueger RF, Balsis S, Skodol AE, Markon KE, Grant BF, Hasin DS (2012) An invariant dimensional liability model of gender differences in mental disorder prevalence: evidence from a national sample. $\mathrm{J}$ Abnorm Psychol 121(1):282-288. doi:10.1037/a0024780

17. Slade T, Watson D (2006) The structure of common DSM-IV and ICD-10 mental disorders in the Australian general population. Psychol Med 36(11):1593-1600. doi:10.1017/S0033291706008452

18. Kessler RC, Ormel J, Petukhova M, McLaughlin KA, Green JG, Russo LJ, Stein DJ, Zaslavsky AM, Aguilar-Gaxiola S, Alonso J, Andrade L, Benjet C, de Girolamo G, de Graaf R, Demyttenaere K, Fayyad J, Haro JM, Hu CY, Karam A, Lee S, Lepine JP, Matchsinger H, Mihaescu-Pintia C, Posada-Villa J, Sagar R, Üstün TB (2011) The development of lifetime comorbidity in the World Health Organization Mental Health Surveys. Arch Gen Psychiatry 68(1):90-100. doi:10.1001/archgenpsychiatry.2010. 180

19. Caspi A, Houts RM, Belsky DW, Goldman-Mellor SJ, Harrington HL, Isreal S, Meier MH, Ramraka S, Shalev I, Poulton R, Moffitt TE (2015) The $\mathrm{p}$ factor: One general psychopathology factor in the structure of psychiatric disorders? Clin Psychol Sci (in press)

20. Eaton NR, Keyes KM, Krueger RF, Noordhof A, Skodol AE, Markon KE, Grant BF, Hasin DS (2013) Ethnicity and psychiatric comorbidity in a national sample: evidence for latent comorbidity factor invariance and connections with disorder prevalence. Soc Psychiatry Psychiatr Epidemiol 48(5):701-710. doi:10.1007/s00127-012-0595-5

21. Kramer MD, Krueger RF, Hicks BM (2008) The role of internalizing and externalizing liability factors in accounting for gender differences in the prevalence of common psychopathological syndromes. Psychol Med 38(1):51-61. doi:10.1017/ S0033291707001572

22. Krueger RF, Chentsova-Dutton YE, Markon KE, Goldberg D, Ormel J (2003) A cross-cultural study of the structure of comorbidity among common psychopathological syndromes in the general health care setting. J Abnorm Psychol 112(3):437-447. doi:10.1037/0021-843X.112.3.437

23. Kendler KS, Aggen SH, Knudsen GP, Røysamb E, Neale MC, Reichborn-Kjennerud $\mathrm{T}$ (2011) The structure of genetic and environmental risk factors for syndromal and subsyndromal common DSM-IV axis I and all axis II disorders. Am J Psychiatry 168(1):29-39. doi:10.1176/appi.ajp.2010.10030340

24. Keyes KM, Eaton NR, Krueger RF, McLaughlin KA, Wall MM, Grant BF, Hasin DS (2012) Childhood maltreatment and the structure of common psychiatric disorders. $\mathrm{Br} \mathrm{J}$ Psychiatry 200(2):107-115. doi:10.1192/bjp.bp.111.093062

25. Eaton NR (2014) Transdiagnostic psychopathology factors and sexual minority mental health: evidence of disparities and associations with minority stressors. Psychology of sexual orientation and gender diversity 1(3):244-254

26. Eaton NR, Krueger RF, Oltmanns TF (2011) Aging and the structure and long-term stability of the internalizing spectrum of personality and psychopathology. Psychol Aging 26(4):987-993. doi:10.1037/a0024406

27. Hicks BM, Blonigen DM, Kramer MD, Krueger RF, Patrick CJ, Iacono WG, McGue M (2007) Gender differences and developmental change in externalizing disorders from late adolescence to early adulthood: a longitudinal twin study. J Abnorm Psychol 116(3):433-447. doi:10.1037/0021-843X.116.3.433

28. Eaton NR, Krueger RF, Markon KE, Keyes KM, Skodol AE, Wall M, Hasin DS, Grant BF (2013) The structure and predictive validity of the internalizing disorders. J Abnorm Psychol 122(1):86-92. doi:10.1037/a0029598

29. Griffith JW, Zinbarg RE, Craske MG, Mineka S, Rose RD, Waters AM, Sutton JM (2010) Neuroticism as a common dimension in the internalizing disorders. Psychol Med 40(7):1125-1136. doi:10.1017/S0033291709991449

30. Watson D, Naragon-Gainey K (2014) Personality, emotions, and the emotional disorders. Clin Psychol Sci 2(4):422-442. doi:10. $1177 / 2167702614536162$

31. Barlow DH, Farchione TJ, Fairholme CP, Ellard KK, Boisseau CL, Allen LB, Ehrenreich-May J (2011) The unified protocol for transdiagnostic treatment of emotional disorders: Therapist guide. Oxford University Press, New York

32. Barlow DH, Sauer-Zavala S, Carl JR, Bullis JR, Ellard KK (2014) The nature, diagnosis, and treatment of neuroticism: back to the future. Clin Psychol Sci 2(3):344-365. doi:10.1177/2167702613505532

33. Cramer AOJ, Waldorp LJ, van der Maas HLJ, Borsboom D (2010) Comorbidity: a network perspective. Behav Brain Sci $33: 137-150$

34. Borsboom D, Cramer AOJ (2013) Network analysis: an integrative approach to the structure of psychopathology. Annu Rev Clin Psychol 9:91-121

35. Borsboom D, Cramer AOJ, Schmittmann VD, Epskamp S, Waldorp LJ (2011) The small world of psychopathology. PLoS ONE. doi:10.1371/journal.pone.0027407

36. Bornstein RF (2010) The rocky road from Axis I to Axis II: extending the network model of diagnostic comorbidity to personality pathology. Behav Brain Sci 33:151-152

37. Cervone D (2010) Aligning psychological assessment with psychological science. Behav Brain Sci 33:152-153

38. Fleeson W, Furr RM, Arnold EM (2010) An agenda for symptom-based research. Behav Brain Sci 33:157

39. Danks D, Fancsali S, Glymour C, Scheines R (2010) Comorbid science. Behav Brain Sci 33:153-155

40. Belzung C, de Villemeur EB, Lemoine M, Camus V (2010) Latent variables and the network perspective. Behav Brain Sci 33:150-151

41. Krueger RF, DeYoung CG, Markon KE (2010) Toward scientifically useful quantitative models of psychopathology: the importance of a comparative approach. Behav Brain Sci 33:163-164

42. Molenaar PC (2010) Latent variable models are network models. Behav Brain Sci 33:166

43. Wright AG, Krueger RF, Hobbs MJ, Markon KE, Eaton NR, Slade T (2013) The structure of psychopathology: toward an expanded quantitative empirical model. J Abnorm Psychol 122(1):281-294. doi:10.1037/a0030133

44. Haslam N (2010) Symptom networks and psychiatric categories. Behav Brain Sci 33:158-159

45. Tzur-Bitan D, Meiran N, Shahar G (2010) The importance of modeling comorbidity using an intra-individual, time-series approach. Behav Brain Sci 33:172-173

46. Johnson W, Penke L (2010) The network perspective will help, but is comorbidity the question? Behav Brain Sci 33:162-163 
47. Kessler RC, Cox BJ, Green JG, Ormel J, McLaughlin KA, Merikangas KR, Petukhova M, Pine DS, Russo LJ, Swendsen J, Wittchen HU, Zaslavsky AM (2011) The effects of latent variables in the development of comorbidity among common mental disorders. Depress Anxiety 28(1):29-39. doi:10.1002/da.20760

48. Pearl J (2009) Causality: models, reasoning and inference, 2nd edn. Cambridge University Press, London

49. MacKinnon DP, Fairchild AJ (2009) Current directions in mediation analysis. Curr Dir Psychol Sci 18(1):16-20

50. Imai K, Keele L, Tingley D (2010) A general approach to causal mediation analysis. Psychol Methods 15(4):309-334

51. McGue M, Osler M, Christensen K (2010) Causal inference and observational research: the utility of twins. Perspect Psychol Sci 5(5):546-556

52. Eaton NR, Krueger RF, South SC, Gruenewald TL, Seeman TE, Roberts BW (2012) Genes, environments, personality, and successful aging: toward a comprehensive developmental model in later life. J Gerontol A Biol Sci Med Sci 67A(5):480-488

53. Kendler KS, Neale MC, MacLean CJ, Heath AC, Eaves LJ, Kessler RC (1993) Smoking and major depression: a causal analysis. Arch Gen Psychiatry 50:36-43

54. Markon KE (2010) Modeling psychopathology structure: a symptom-level analysis of Axis I and II disorders. Psychol Med 12(2):273-288. doi:10.1017/S0033291709990183

55. McFarland DJ, Malta LS (2010) Symptoms as latent variables. Behav Brain Sci 33:165-166

56. Box GEP, Draper NR (1987) Empirical model-building and response surfaces. Wiley, New York 